\title{
The Your COVID-19 Risk Assessment Tool and the Accompanying Open Access Data and Materials Repositories
}

Gjalt-Jorn Ygram Peters ${ }^{1 *}$, Dominika Kwasnicka ${ }^{2 *}$, Rik Crutzen ${ }^{3}$, Gill A ten Hoor ${ }^{3}$, Tugce Varol ${ }^{3}$, Mahdi Algargoosh ${ }^{4}$, Eskinder Eshetu Ali ${ }^{5}$, Mudassir Anwar ${ }^{6}$, Sali Rahadi Asih ${ }^{7}$, Zuhal Feryal Baltas ${ }^{8}$, Emma

Berry ${ }^{9}$, Kebede Abera Beyene ${ }^{10}$, Katarzyna Anna Campbell ${ }^{11}$, Bruno Moreira Carneiro ${ }^{12}$, Laura

Castillo-Eito ${ }^{13}$, Amy Hai Yan Chan ${ }^{14}$, Sabrina Cipolletta ${ }^{15}$, Ann DeSmet ${ }^{16}$, Triana Kesuma Dewi17, Alexandra Lelia Dima ${ }^{18}$, Jorge Encantado ${ }^{19}$, Tracy Epton ${ }^{20}$, João Melo Figueiredo ${ }^{21}$, Gustavo DalCin

Fracarolii22, Gebremedhin Beedemariam Gebretekle²3, Pierre Gérain ${ }^{24}$, Cristina Albuquerque Godinho $^{25}$, Lisa Graham-Wisener', James A. Green ${ }^{26}$, Jenny M Groarke ${ }^{27}$, Thomas Gültzow ${ }^{28}$, Elif Basak Guven ${ }^{29}$, Roel C.J. Hermans ${ }^{3}$, Jennifer Inauen ${ }^{30}$, Angelos P. Kassianos ${ }^{31}$, Tatiana Valerievna Kazantseva ${ }^{32}$, Els Keyaerts ${ }^{33}$, Laura Maria König ${ }^{34}$, Daniela Lange ${ }^{35}$, Emelien Lauwerier ${ }^{36}$, Yongchan $\mathrm{Lie}^{3}$, Andrian Liem ${ }^{37}$, Aleksandra Luszczynska ${ }^{2}$, Marta M. Marques ${ }^{38}$, Hannah Catherine Moore ${ }^{39}$, Chris Noone ${ }^{40}$, Johanna Nurmi ${ }^{41}$, Ratri Nurwanti ${ }^{42}$, Elif Suna Ozbay ${ }^{43}$, Iga Palacz-Poborczyk2 ${ }^{2}$, Rebecca Anne Pedruzzi ${ }^{44}$, Louise Poppe ${ }^{36}$, Lucy Mabel Porter ${ }^{45}$, Daniel Powell ${ }^{46}$, Bruna Salati Nani Rinaldi ${ }^{47}$, Alexis Ruffault ${ }^{48}$, Carsten Schmitz ${ }^{49}$, Urte Scholz ${ }^{50}$, Ana-Maria Schweitzer ${ }^{51}$, Yasemin Selekoğlu Ok ${ }^{52}$, Medha Shree ${ }^{53}$, Yasinta Astin Sokang54, Mei Yee Tang55, Silvia Caterina Maria Tomaino ${ }^{15}$, Stefan Verweij $^{56}$, Stan Vluggen ${ }^{3}$, Lisa Marie Warner ${ }^{57}$, Rochelle E Watkins ${ }^{58}$, Szilvia Zörgö ${ }^{59}$ \& Sylvia Roozen ${ }^{60}$

This is a preprint - it has not been peer-reviewed.

${ }^{1}$ Open University of the Netherlands, Netherlands

2 SWPS University of Social Sciences and Humanities, Poland

${ }^{3}$ Maastricht University, Netherlands

${ }^{4}$ The University of Auckland, New Zealand

${ }^{5}$ Addis Ababa University, Ethiopia

${ }^{6}$ University of Otago, New Zealand

${ }^{7}$ Faculty of Psychology, Universitas Indonesia, Indonesia

${ }^{8}$ Cerrahpaşa medical faculty, Turkey

${ }^{9}$ Queen's University Belfast, United Kingdom

${ }^{10}$ School of Pharmacy, the University of Auckland, New Zealand

${ }^{11}$ University of Nottingham, United Kingdom

${ }^{12}$ Universidade Federal de Rondonópolis, Brazil

${ }^{13}$ University of Gloucestershire, United Kingdom

${ }^{14}$ University of Auckland, New Zealand

${ }^{15}$ University of Padua, Italy

${ }^{16}$ Université libre de Bruxelles, Belgium

${ }^{17}$ Faculty of Psychology, Airlangga University, Indonesia

${ }^{18}$ Research on Healthcare Performance RESHAPE, INSERM U1290,

Université Claude Bernard Lyon 1, France

${ }^{19}$ Faculdade de Motricidade Humana, Universidade de Lisboa,

Portugal

${ }^{20}$ University of Manchester, United Kingdom

${ }^{21}$ Freelancer, Portugal

22 SAST - Segurança e Saúde do Trabalho, Brazil

${ }^{23}$ Institute of Health Policy, Management and Evaluation, University

of Toronto, Canada

${ }^{24}$ SCALab UMR CNRS 9193 - University of Lille, France

${ }^{25}$ Universidade Católica Portuguesa, Católica Research Centre for

Psychological - Family and Social Wellbeing, Portugal

${ }^{26}$ University of Limerick, Ireland

${ }^{27}$ Queen's University Belfast, Ireland

${ }^{28}$ CAPHRI Care and Public Health Research Institute, Department of

Health Promotion, Maastricht University, Maastricht, the

Netherlands, Netherlands

${ }^{29}$ Istanbul Technical University, Turkey

${ }^{30}$ University of Bern, Switzerland
${ }^{31} 1$. University of Cyprus, Department of Psychology / 2. University College London, Department of Applied Health Research, Cyprus

${ }^{32}$ Saint-Petersburg State University, Russian Federation

${ }^{33}$ UZ Leuve, Belgium

${ }^{34}$ University of Bayreuth, Germany

${ }^{35}$ Medical School Berlin, Germany

${ }^{36}$ Ghent University, Belgium

${ }^{37}$ University of Macau, Macau

${ }^{38} 1$ - Trinity College Dublin, Ireland; 2- Comprehensive Health

Research Centre, NOVA Medical School, Lisbon, Portugal, Portugal ${ }^{39}$ Wesfarmers Centre of Vaccines and Infectious Diseases, Telethon

Kids Institute, University of Western Australia, Australia

${ }^{40}$ NUI Galway, Ireland

${ }^{41}$ University of Helsinki; University of Cambridge, Finland

${ }^{42}$ Brawijaya University, Indonesia

${ }^{43}$ Bilkent University, Turkey

${ }^{44}$ Telethon Kids Institute, Australia

${ }^{45}$ University of Exeter, United Kingdom

${ }^{46}$ University of Aberdeen, United Kingdom

${ }^{47}$ Holy House of Mercy of Piracicaba / Caterpillar Inc Brazil, Brazil

${ }^{48}$ French Institute of Sport, France

${ }^{49}$ LimeSurvey GmbH, Germany

${ }^{50}$ University of Zurich, Switzerland

${ }^{51}$ FUNDATIA BAYLOR MAREA NEAGRA, Romania

${ }^{52}$ Dokuz Eylül University Faculty of Nursing, Turkey

${ }^{53}$ Viztar International, India

${ }^{54}$ Krida Wacana Christian University, Indonesia

${ }_{55}^{5}$ Newcastle University, United Kingdom

${ }^{56}$ Evently, Netherlands

${ }^{57}$ MSB Medical School Berlin, Germany

${ }^{58}$ Telethon Kids Institute, The University of Western Australia,

Australia

${ }^{59}$ Semmelweis University, Hungary

${ }^{60}$ Governor Kremers Centre-Department of Health Promotion

Maastricht University, Netherlands

* Shared first authorship 


\begin{abstract}
(150 words max)
In March 2020, the Your COVID-19 Risk tool was developed in response to the global spread of SARS-CoV-2. The tool is an online resource based on key behavioural evidencebased risk factors related to contracting and spreading SARS-CoV-2. This article describes the development of the tool, the produced resources, the associated open repository, and initial results. This tool was developed by a multidisciplinary research team consisting of more than 150 international experts. This project leverages knowledge obtained in behavioural science, aiming to promote behaviour change by assessing risk and supporting individuals completing the assessment tool to protect themselves and others from infection. To enable iterative improvements of the tool, tool users can optionally answer questions about behavioural determinants. The data and results are openly shared to support governments and health agencies developing behaviour change interventions. Over 60000 users in more than 150 countries have assessed their risk and provided data.
\end{abstract}

\title{
Keywords
}

health behaviour change, impact, risk, people, strategies, systems, COVID-19 
The COVID-19 outbreak has substantially increased the burden on healthcare systems through increased primary care, ambulance and emergency department presentations due to the virus itself and through indirect mechanisms such as increasing rates of poor mental health ${ }^{1}$. In the absence of widely available treatments and vaccines (which only became available to most countries in 2021), rapidly rising prevalence and mortality rates have forced most countries to introduce several reforms such as lockdowns to slow down the virus spread. Such measures have substantially disrupted social and economic systems around the world, globally impacting almost all countries and territories ${ }^{2,3}$.

Given the crucial role of human behaviour in virus spread ${ }^{4}$, most countries introduced preventive measures that require behaviour change, including social distancing, wearing masks, and increasing the frequency of hand washing 5 . Globally, governments and health agencies have worked collaboratively with social scientists to influence human behaviour to slow down and to contain the virus spread ${ }^{5,6}$. The effectiveness of these behavioural policies has varied across countries and territories, with some achieving great successes and others facing the challenges of new outbreaks ${ }^{7}$.

Following the principles of Open Science ${ }^{8}$, global cooperation, planning and effective governance ${ }^{9}$, scientists involved in this project joined in efforts to find solutions to prevent and contain the spread of SARS-CoV-2 ${ }^{10}$. The 'Your COVID-19 risk' project commenced in March 2020, before SARS-CoV-2 vaccines were available, and when the effectiveness of preventive measures were under debate. The project employed several technologies and methods to (1) facilitate the use of behavioural science solutions in a systematic manner, (2) develop and implement a tool that assesses behaviour-related COVID-19 risk, (3) provide instant risk estimates and suggestions for behaviour change, and (4) share the data with 
researchers, governments and health agencies to inform future policy and practice during the pandemic.

The Your Covid-19 risk tool consists of ten questions informed by a rapid systematic assessment of the literature addressing the risk factors for contracting and spreading SARSCoV-2, and two expert consultations. We leveraged Acyclic Behaviour Change Diagrams ${ }^{11}$ (ABCDs) to develop and make transparent a behaviour change intervention that tool users received based on their answers to the questions. Behavioural determinants (specifically, psychological constructs defined in the Reasoned Action Approach, RAA ${ }^{12}$ ) were specified using Decentralized Construct Taxonomies ${ }^{13}$ (DCTs) to facilitate standardisation of construct definitions and corresponding measurement instruments across countries and contexts. Questions measuring these determinants were integrated into the tool, and the results of these questions were analysed using Confidence Interval-Based Estimation of Relevance (CIBER) plots to identify the most important determinants ${ }^{14,15}$.

The Open nature of this tool means that it lends itself well to adaptation to other situations and behaviours to address the SARS-CoV-2-related challenges of the coming months ${ }^{16}$, as well as other, unrelated, behaviours. In the results section, we first describe the tool itself (the main result) and provide preliminary guidance on adapting the tool, and using and describing the produced data. In the Discussion section, we briefly describe the tool, and discuss the strengths and limitations. Finally, in the Methods section, we will describe the development process and the empirical, theoretical, and operational underpinnings of the Your COVID-19 Risk tool. 


\section{Results}

\section{The Your COVID-19 Risk tool}

The tool, accessible through https://your-covid-19-risk.com, consists of two parts: a static website and a LimeSurvey ${ }^{17}$ implementation. The tool is optimised for mobile devices, because many people primarily access the internet using mobile phones ${ }^{18-20}$, does not store any personal data, and can be used repeatedly. We created a static website (instead of a website using server-side parsed code in, e.g., PHP, Python, or Java) to facilitate free hosting, eliminate the need to implement custom configurations of the webserver, and facilitate adaptation of the website. All content was translated from English into 21 additional languages (yielding a total of 22 languages) by the international experts participating in the project (for most languages forward and back translations were used). The included languages are: Amharic, Arabic, Chinese (simplified and traditional), Dutch, English, French, German, Greek, Hebrew, Hungarian, Indonesian, Italian, Japanese, Korean, Polish, Portuguese (Brazilian and Portuguese), Romanian, Spanish, Turkish and Urdu.

The static Your COVID-19 Risk tool site consists of a homepage, details about the project and project volunteers, and pages briefly describing the underlying theory, risk model, and data management. The home page features a button labelled 'Find out your risk!' that links to LimeSurvey, where tool users answer ten Risk Estimation Questions (see below) before being sent back to the results page on the static site, which shows them their estimate and the behaviour change intervention.

The Risk Estimation Questions assess users' location (country and for some countries also region), age, and gender. The users are then asked to select details about their work environment (i.e., whether they work with patients and vulnerable groups; with children; 
with co-workers; with the general public; or from home or not working). Users are also asked about day-to-day behaviours they currently perform (including leaving the house for food, healthcare, exercise, visiting family, friends etc - multiple options can be selected), and the conditions under which these day-to-day behaviours would change (e.g., if they had COVID-19 symptoms, were in contact with someone who is sick or showing symptoms, or if their government imposed further restrictions). Additionally, tool users are asked to identify which of their day-to-day behaviours would change under such conditions (e.g., I would stop visiting family). Next, the users are asked to estimate how often they keep their distance (specifically $1.5 \mathrm{~m}$ ) from others in public, identify the situations in which they regularly wash or clean their hands and the procedures they follow to do so.

Recommendations for wearing masks were not clearly defined in March 2020, when there was still concern for supplies for healthcare workers, and limited information on or availability of cloth masks. There was active discussion around whether we should include wearing masks in the tool, and we ultimately decided to forgo it in this first version of the tool.

In the penultimate stage of using the tool, users can opt in to answer additional questions (between 0 and 20 Determinant Mapping Questions; see Methods) in order to improve the tool. Once they responded to these questions, the survey produces a string encoding the risk estimate and personalised intervention content before redirecting users to their results page on the static site. The results page describes and illustrates the individual user's 'risk estimate' and behaviour change intervention (a selection of tailored messages labelled their 'safety estimate;' see Methods section). 


\section{Adapting the Your COVID-19 Risk Tool}

Adapting the tool requires some familiarity with the technologies used to build the tool such as Git, R and R Markdown, Hypertext Markup Language (HTML), Cascading Stylesheets (CSS), and JavaScript (also see the Methods section). To adapt the tool, the Git repository hosted at https://gitlab.com/a-bc/your-covid-19-risk can be cloned or forked. The static site is located in the 'v1/website' directory, and loads almost all content from JavaScript Object Notation (JSON) files in the 'json' subdirectory and from ResourceBundle files in the ' $\mathrm{i} 18 \mathrm{n}$ ' subdirectory (' $\mathrm{i} 18 \mathrm{n}$ ' is a numeronym that stands for 'internationalisation'). The survey questionnaires are located in the 'operationalizations/limesurvey' directory: these are tab-separated files in the .txt format that can be imported directly into LimeSurvey. The project relies heavily upon a number of R scripts (see the Methods section) which read data from spreadsheets stored in Google Sheets, create local backups in Microsoft Excel's .xlsx format, and parse the data to produce the resources that inform the static site and the LimeSurvey part of the tool (see the Methods section for details). These scripts are available in the 'v1/scripts' directory.

\section{Using the Your COVID-19 Risk Data}

The Your COVID-19 Risk data are available in a different dedicated Git repository that is hosted at https://gitlab.com/a-bc/your-covid-19-risk-data. This repository contains a series of comma-separated values (CSV) files, as well as file manifests in several formats and an R script to import and merge all data. Whenever the data pipeline is run, these files are updated. It is also possible to inspect the analysis files, which are available in the project's main repository in the ' $\mathrm{v} 1 /$ scripts' directory. 


\section{Using the analysis file for the Your COVID-19 Risk Project}

The Your COVID-19 Risk project contains an R Markdown file that analyses the data produced by the tool. The rendered version of this file is hosted by GitLab Pages at https://abc.gitlab.io/your-covid-19-risk/v1/results/analyses/. In this section, we use brief summaries of the results of these analyses to guide readers through the sections of that file. These results pertain to the full data set (i.e., for all countries combined), and as such, give a good impression of the tool users, but not of any country, global patterns, or specific timeframe.

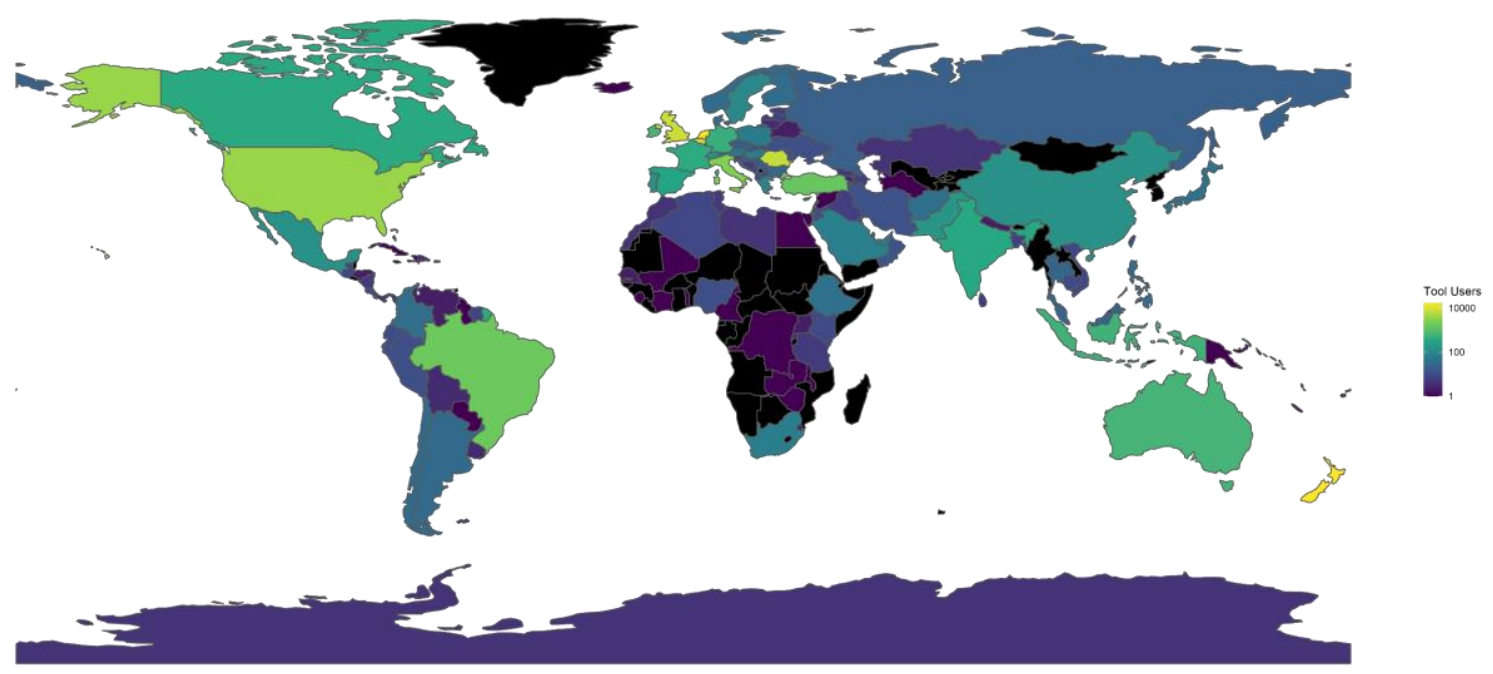

Figure 1: A world map showing the number of tool users across countries.

\section{Countries}

The tool first launched in the Netherlands on May 7th, 2020 and remaining countries launched using a staggered approach over the following month. From this date until June the $9^{\text {th }}$ of 2021, 102909 individuals from 166 countries used the Your COVID-19 Risk tool (see the world map of tool users in Figure 1), 63850 (from 153 countries) of whom completed the full assessment. Of the remaining 39 059, 27424 solely opened the first page, and 11635 started but did not complete the risk assessment and did not receive behavioural support. 
This equates to a $15 \%$ drop-out over all countries. The following countries had 100 or more users who completed the tool: Netherlands ( $n=15$ 376), New Zealand ( $n=14$ 615), Belgium ( $n=7$ 052), United Kingdom ( $n=6$ 978), Romania ( $n=6$ 187), United States $(n=2998)$, Italy $(n=2$ 240), Brasil ( $\mathrm{n}=1415)$, Turkey ( $\mathrm{n}=1$ 137), Ireland (Republic) $(\mathrm{n}=587)$, Australia $(\mathrm{n}=523)$, Indonesia $(n=440)$, Germany $(n=385)$, France $(n=340)$, Canada $(n=323)$, Spain $(n=281)$, India $(\mathrm{n}=251)$, Cyprus ( $\mathrm{n}=201)$, Portugal $(\mathrm{n}=117)$, Switzerland $(\mathrm{n}=112)$, Mexico $(\mathrm{n}=109)$, China $(\mathrm{n}=104)$, and Pakistan $(\mathrm{n}=101)$. Tool use over time was strongly related to local promotional activities. Use increased rapidly following the tool launch in May-June 2020 with a few spikes in use during July.

\section{Risk Estimation Questions}

Tool users reported their age as follows: $40-49$ years (20.1\%), 50-59 years (19.5\%), 30-39 years $(19.4 \%)$, 20-29 years (17.6\%), 60-69 years (14.3\%), and 8.9\% selected they were younger than 20 years or older than 70 years. The remaining $0.1 \%$ declined to disclose their age. Most users identified as female (54.1\%) or male (45.2\%), with $0.31 \%$ identifying as nonbinary, third gender, or a self-specified gender.

In response to the (multiple choice) questions about work environment, most tool users indicated they were working from home or not working (63.1\%), and $28.2 \%$ indicated they were (instead or also) professionally in contact with the public (e.g., in the hospitality or public transport sectors). A further $6.7 \%$ indicated they were professionally in contact with patients (e.g., at hospitals, medical centres, or nursing homes), and $6.1 \%$ indicated they were professionally in contact with children (e.g., at childcare, primary, or secondary education). 
In response to the (multiple choice) questions about day-to-day activities, most tool users indicated they were leaving the home for food, medicine, or healthcare (75.8\%), walking or exercising outdoors (70.1\%), and/or leaving the home for other shops and services (52.2\%). Almost half of tool users (45.1\%) indicated they were mostly staying at home, while many (35.9\%) indicated they were visiting family, and/or visiting friends (27.1\%). The most frequently identified reasons to change these day-to-activities included having symptoms of COVID-19 (85.4\%), contact with a sick person or a person who showed symptoms $(73.3 \%)$, and government restrictions (65.8\%). Only $4.4 \%$ indicated they would not change their day-to-day activities while $0.4 \%$ specified other reasons

When asked what day-to-day activities would change as a result of such conditions, most tool users indicated they would stop leaving the house for other shops or services (43.4\%), and/or for food, medicine, or healthcare (40.6\%). A further 34.6\% indicated they would cease outdoor walks or exercise, $31.9 \%$ indicated they would cease visiting family, and $25 \%$ indicated they would cease visiting friends. The minority $(3.2 \%)$ specified other reasons.

When asked to estimate how often they keep their distance (specifically 1.5 metres) from others in public, $0.9 \%$ indicated they did this (almost) never; $1.9 \%$ selected rarely; $8.7 \%$ sometimes; $23.6 \%$ often; $61.5 \%$ (almost) always; and 3.5\% indicated they were never in public places.

Most (83.4\%) tool users indicated they wash their hands after touching public or frequently touched surfaces (e.g., doorknobs, railings, tables, buttons/switches), and /or on entering their home, place of work, or other building they will be spending some time in (77.4\%). Over three-quarters $(76.6 \%)$ indicated they wash their hands before they handle or eat food, and/or after they have been in direct contact with someone else (65.2), after they 
sneeze or cough $(41 \%)$, and before or after they have touched their face, nose, or mouth (28.9\%). Only $3.9 \%$ indicated they never or only occasionally wash/clean their hands.

Almost all (91.1\%) tool users indicated they normally use soap or hand sanitiser in their hand washing procedure, while $69.8 \%$ indicated that they wash for at least 20 seconds. Of the tool users $47 \%$ identified that they clean under their nails and between their fingers, and $1.2 \%$ indicated they do not do any of these procedures.

\section{Determinant Mapping Questions}

Based on users' answers to the Determinant Mapping Questions (which in this case concerned following their country-specific guidelines for social distancing behaviours, see the Methods section), Confidence Interval-Based Estimation of Relevance (CIBER) plots were created ${ }^{14,15}$. The results of these plots allow selection of the most relevant determinants reflecting relevant theoretical, methodological, and statistical considerations and are available (for each country with enough data) under the Attitude, Perceived Norm, and Perceived Behavioural Control tabs. An example CIBER plot is shown in Figure 2, where a selection of five sub-determinants is illustrated: three that were identified as important and two that were not important. In short, the left panel shows each (sub)determinant's raw data (i.e., the distribution of sample scores) as well as the $99.99 \%$ confidence interval for the mean. The right panel shows the $95 \%$ confidence interval of the association with one or more targets - in this specific case, the bivariate correlation with tool users' self-reported social distancing as measured by one of the Risk Estimation Questions. Whether a subdeterminant is selected as an intervention target normally depends on whether it is bivariately associated with behaviour (or a proxy of behaviour) and on its univariate distribution (indicating room for improvement). High correlations can be driven by only a 
few data points so it is possible for sub-determinants that barely have any room to improve to still be associated with the behaviour ${ }^{14}$.

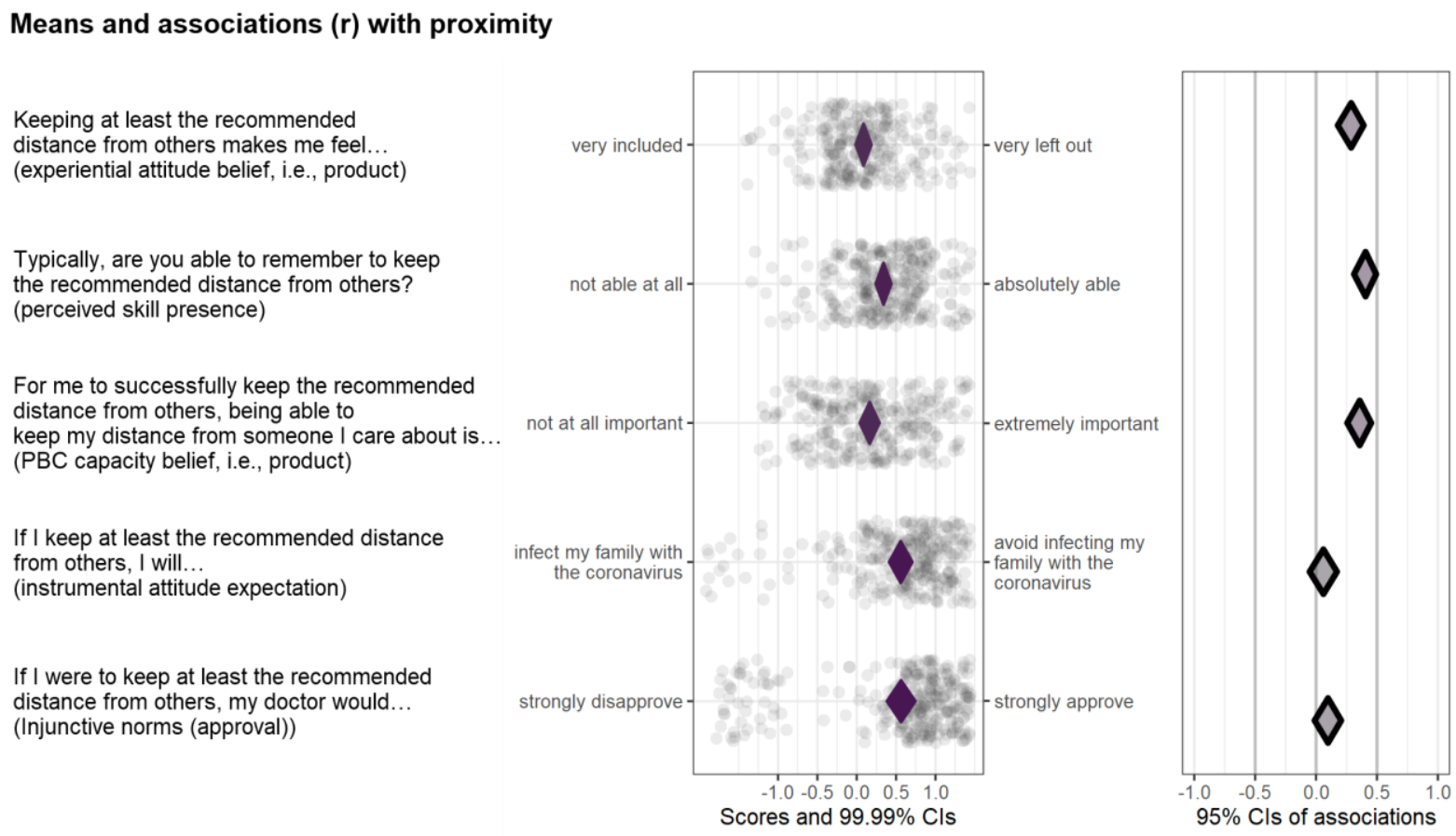

Figure 2: An example Confidence Interval-Based Estimation of Relevance (CIBER) plot, showing three relevant and two irrelevant determinants in the Dutch subsample.

In addition to the CIBER plots, overviews of all sub-determinants are provided in tables in the 'Overview' tab. Three overviews are produced: the last one is sorted by the bivariate correlation of each sub-determinant with the target behaviour, and the first two are sorted by two versions of the Potential for Change index ( $\mathrm{P} \Delta$ ), which is a quantitative summary of the information in the CIBER plots. The original P $\Delta$ was conceptualised to optimise intervention tailoring and improve the prediction of individual-level intervention effectiveness ${ }^{21}$. For this project, two sample-level variants were computed to facilitate subdeterminant selection.

The first, labelled the Potential for Change Index 1 (P $\Delta 1)$, was computed as follows: for sub-determinants with a positive zero-order correlation with behaviour, the sample mean was subtracted from the observed maximum score, and the result was multiplied by the 
zero-order correlation. For sub-determinants with a negative zero-order correlation with behaviour, the sample mean was subtracted from the observed minimum score and the result was multiplied by the zero-order correlation.

The second, labelled the Potential for Change Index 2 (P $\Delta 2)$, was computed as follows: for sub-determinants with a positive zero-order correlation with behaviour, the sample mean was subtracted from the .95 quantile of the scores, and the result was multiplied by the squared zero-order correlation (i.e., the proportion of explained variance). For subdeterminants with a negative zero-order correlation with behaviour, the sample mean was subtracted from the .05 quantile of the scores, and the result was multiplied by the squared zero-order correlation (i.e., the proportion of explained variance). The second variant effectively takes the $5 \%$ trimmed maximum and minimum, rendering it less sensitive to outliers, penalises weak associations with behaviour more severely and decreases sensitivity to differences between correlations. These differences usually render the second variant more robust over different samples.

\section{Discussion}

The real-world problem this tool aimed to ameliorate is the spread of the SARS-CoV-2 virus. The main aim of creating this tool was to support users to estimate their infection risk, provide them with strategies to minimise risk and gather data to inform future health promotion interventions and policy during the pandemic. The tool users received their Risk Estimate and their Safety Estimate. Both were optimised for the tool users' situation based on their unique responses. The Safety Estimate was developed using Acyclic Behaviour Change Diagrams (ABCDs) and the Determinant Mapping Questions were used to create 
Confidence Interval-Based Estimation of Relevance (CIBER) plots to subsequently inform policy and practice initiatives regarding prevention efforts.

A similar tool that aims to assess COVID-19 risk and provide feedback to users has been developed by the World Health Organization ${ }^{1}$. This tool asks specific questions relevant to protective and risk-relevant behaviours and after each question, gives feedback in relation to that behaviour. For example, tool users are asked about shaking hands and are complemented if following best practices. If users indicate they do not follow best practices then they are presented with suggestions on how to improve their behaviour. Like the tool presented here, aims of The WHO tool include to inform decision making, support users to protect themselves and others from infection, and to promote best behavioural practices. The WHO tool was also implemented using open-source LimeSurvey software. A key difference is the number of available languages (23 versus 6). In addition, the information provided by the WHO tool includes main guidelines that are informative for people who were not aware of best practices (keeping distance, hand washing, self-isolating if and when needed) but may not be useful to users who were already following best practices; as they may wish to receive more sophisticated and nuanced risk estimates and recommendations. Compared to our tool, it is likely that the WHO had more (i.e., any) financial backing. Finally, we could not identify the theoretical and evidence bases of the WHO tool: it is not clear which (sub-)determinants they target with which messages. Our assessment suggests that the tool only targets knowledge, as opposed to norms, self-efficacy, or other determinants, but we cannot verify this. In comparison, because the tool we present and all underlying materials are open access and open-source, it lends itself well to adaptation by

\footnotetext{
${ }^{1}$ See https://extranet.who.int/dataformv3/index.php/641777?newtest=Y\&lang=en
} 
other researchers and organisations. Our tool is transparent in its design and precise in terms of estimating risk and providing tailored behaviour change recommendations. Finally, the data that are gathered are made publicly available so they can be used by the research community and subsequently by practitioners and policy makers who are responding to current and future pandemics. Tools such as these are needed to support the community in their guideline adherence. However, they also suffer some limitations.

There were two important limitations to the present project. The first limitation concerns the spontaneous inception of the project and limited (financial) resources available, including no pre-existing plan for sustainability. The project was initiated by a large group of volunteers from all over the world who responded to a public health emergency of international concern in urgent need of international coordinated efforts. As the project progressed, volunteers' time became more limited. This was also the case for the small number of volunteers that the project depended on for coordination and production of the mutually aligned instructions. The second limitation was that the interlinking set of software applications used for this project each required specific expertise to connect and manage. This expertise was limited in the team, creating a bottleneck in project progress.

The solution to both limitations is relatively straightforward: funding. However, funding calls often target either research or software development. This was not a research project and did not collect data to answer research questions. Redesigning it to become research is possible but would introduce considerable delays (requiring, e.g., crossculturally validated measures and ethical approvals). Similarly, this project did not develop innovative software solutions, but instead combined and used existing applications. Instead of being research or software development, this project's strength lies in the rigorous application of cutting-edge behaviour change science, combined with a theory- and 
evidence-based risk model, in a well-designed online application. This unique profile does not lend itself to conventional funding channels, and without the support of volunteers would never be developed in such a short timeframe. As such the resource presented in this article is unique.

This tool has several strengths. First, all materials, source code, and design documents are publicly available and align with open science principles to enhance transparency and facilitate adaptation to other contexts or behaviours. Second, because the underlying behaviour change theory and assumptions are described in detailed ABCDs and freely available, it is possible for others to improve the intervention based on new insights about determinant structures. Third, this tool is based on behaviour change theory and evidence and has the potential to further inform science through the open data that are gathered. Furthermore, behaviour change researchers and practitioners can interpret the results and use these to inform practice and policy. Finally, by being multi-lingual, this tool is available to communities all over the world, and through its collaborative development considers contexts from many cultures and regions, with good representation of the Global South. Similarly, the multi-disciplinary nature of the group of volunteers ensured application of a wide array of best practices across different disciplines, such as behavioural science, virology, epidemiology, and public health, which allowed us to respect each discipline's limitations (e.g., the behaviour change intervention was based on psychology, but the risk model was based on virology and epidemiology).

In this publication, we have described the process and systematic method used to curate a tool to assess COVID-19 risk, and provide risk and safety estimates accompanied by persuasive messages. The tool was designed by more than 150 experts including behavioural scientists, psychologists, virologists, methods experts, representatives from the 
government, consumers and other groups. All contributing specialists worked free of charge in a coordinated effort to develop an evidence-based tool that allows gathering of Open Source data to inform future interventions and policies. The database is also open to other researchers who are interested in answering specific research questions related to COVID19. To move behavioural science forward, and to effectively respond to the current pandemic, international coordinated efforts are needed. Here, we have presented a tool that has the potential to contribute to slowing down SARS-CoV-2 virus spread informing researchers and practitioners about current behaviours and psychology underpinning this situation.

\section{Methods}

\section{Project Overview}

This project applied insights from behaviour change science to develop, implement, and disseminate an online tool to help contain the COVID-19 pandemic for communities worldwide. This tool allows the user to assess the 'additional risk' of contracting and spreading the virus leading to COVID-19. Additional risk involves situations and behaviours that the individual has some form of control over- and can do something about. The tool does not account for risks related to underlying health conditions. The tool development followed a rigorous, systematic, and scalable approach. We (i.e., the group of volunteers who developed this tool) used theory and already existing empirical results. The tool was created in ten weeks, in a worldwide, largely decentralised collaboration of over 150 experts (for

reflections on this process, see $^{9}$ ). Note that the tool was not built to test theory, hypotheses, or answer research questions, but to leverage behaviour change theory and evidence to 
support people in their behaviour change efforts. Responding to the current COVID-19 pandemic, the aim of this project was three-fold:

1. provide a widely available tool that allows people to get an understanding of their COVID-19-related risk, both to themselves and to others;

2. apply behaviour change theory to present people with persuasive messages that help decrease their risk;

3. explore tool users' COVID-19-related behaviours and related determinants to enable improvements to the tool and recommend avenues for interventions and policy to governments, policymakers, and prevention and health promotion organisations.

To provide information about COVID-19-related risk, we first required a risk model that would estimate risk based on the unique situations of tool users.

\section{The Risk Model}

The Risk Model was established in two expert consultations. The main goal of the first consultation was to determine the most prominent risk behaviours and risk factors. In order to provide tool users with an engaging tool, and to keep the number of questions minimal; we aimed to identify key behavioural factors that are within the users' control and can be addressed through behaviour change principles. In the second expert consultation, we then determined the degree to which each risk behaviour and risk factor contributed to the overall risk estimate.

\section{Expert Consultation 1}

Scientific articles on COVID-19 $(n=110)$ were reviewed to determine key risks that were associated with SARS-CoV-2 infection. The list of risks was firstly categorised into 
behaviour type for (1a) risk behaviours for contracting the virus (i.e., hygiene behaviours, social interaction, transport and travel behaviours, health related behaviours), and (1b) risk behaviours for spreading the virus to others. Overlap between these categories was expected. Secondly, the list of risks was also categorised into other risk characteristics: (2a) other indicators of risk of contracting and spreading the virus (i.e., indicators related to profession, living situation) and (2b) other indicators of risk for developing severe conditions (i.e., general indicators such as health status).

The list of risk behaviours and risk characteristics were then presented to 10 virologists and epidemiologists to assess how important these factors were for spreading the SARSCoV-2 virus. The results were collated and also ranked by specificity (i.e., how many people would be affected). A list of key behaviours and characteristics was defined. Then, vignettes were also assessed with scenarios spanning a range of nationalities and situations illustrating how the virus could be spread. The putative key behaviours and characteristics were formulated into risk estimate questions and were answered by experts from the point of view of the person described in the vignettes to ensure they encompassed a range of situations.

\section{Expert Consultation 2}

In the final tool, the answers to the questions about the key behaviours and risk factors were equated to a level of risk. The risk provided the user with a category of risk. Categories were commencing at 'standard' and had four categories in total. A survey was created to determine which level of risk (standard to exponential; 1-4) an individual would be at for each of the assessed behaviours and characteristics. Fifty-seven experts including behaviour change specialists $(n=29)$, virologists/epidemiologists $(n=11)$, and medical professionals 
$(n=17)$ were asked to: (a) indicate the level of risk performance / non-performance of the behaviours / characteristics would result in, and (b) their level of confidence in their estimate (low [weighted as .33], medium [.66] or high [1]). For part (a) the experts could use the initial risk estimates provided by virologists and epidemiologists, their own specialist knowledge, advice from other sources (e.g., government guidelines) or the academic literature (indicating the source they used).

The initial results were weighted by confidence and a sensitivity analysis was conducted to determine whether confidence-based weighting influenced the risk estimates. This was not the case because the highest rated risks were the same as the preliminary survey conducted with virologists and epidemiologists. The results did not differ by field or expertise (virologists or epidemiologists vs other professions) or by source of knowledge; therefore, all the data were used in the risk estimate tool.

\section{Designing the Risk Estimate}

The risk estimates were presented in categories and we consulted health literacy experts about the number of categories. Three to five active categories were determined by behaviour change experts to be easily understood by most people. A larger number of categories would be most desirable to track a change in results; however, it was unlikely the tool would be used for this purpose and too many categories can be hard to parse for those with lower literacy or numeracy levels. We wanted to prevent anxiety for those tool users getting the highest level of risk and also prevent a false sense of security for tool users getting the lowest level of risk. Therefore, the tool was designed in such a way that the highest and lowest categories in the visualisation were not mapped onto risk estimate scores, and so could never be highlighted for a tool user (to not induce excessive fear or 
complacency, respectively). We compared the results for five and six categories (of which three and four would be attainable, respectively). The vignettes used in the initial testing of the risk model were adapted (to ensure all questions could be answered) and used again to answer the risk estimate questions. Two additional scenarios were added (introducing a fictional character Minnie - with the minimal possible risk and Max - with the maximum possible risk) to give the full range of risk scores. Each of the vignettes was scored by two people and the scores on the research estimate questions corresponded to our qualitative assessment of the people in the vignettes. We established that four attainable categories, for a total of six risk levels were suitable.

The risk estimate was determined to be due to three factors: 'risk of getting the virus' (i.e., through proximity or lack of social isolation), 'risk from not removing the virus' (i.e., through lack of handwashing) and other uncontrollable factors (e.g., demographics). The demographic factors (i.e., country, gender, age, occupation) were not controllable, although could affect individuals' personal risk so the messages were to be presented as 'protect yourself' (i.e., if you were at high risk) or 'protect others' (i.e., if you were at low risk). A visual image combining these three factors was considered appropriate to communicate the risk level (e.g., an avatar of someone surrounded by virus) and a speed gauge for the demographic factors to show the reason for changing behaviour. The design choices were discussed within the larger team of volunteers and it was decided that one image for each type of risk was to be presented with a scale to indicate risk category (Figure 3). Six categories were agreed upon (4 active) and captions and text written for each. The proposed materials were piloted with general public representatives to ensure that they were easy to understand and interpret. The materials were adjusted in line with the received feedback. 
After tool users received their risk estimate, they were presented with what we called the 'Safety Estimate', which was a tailored minimal behaviour change intervention aiming to support people in three behaviours: keeping sufficient distance from others, self-isolation, and hand hygiene.

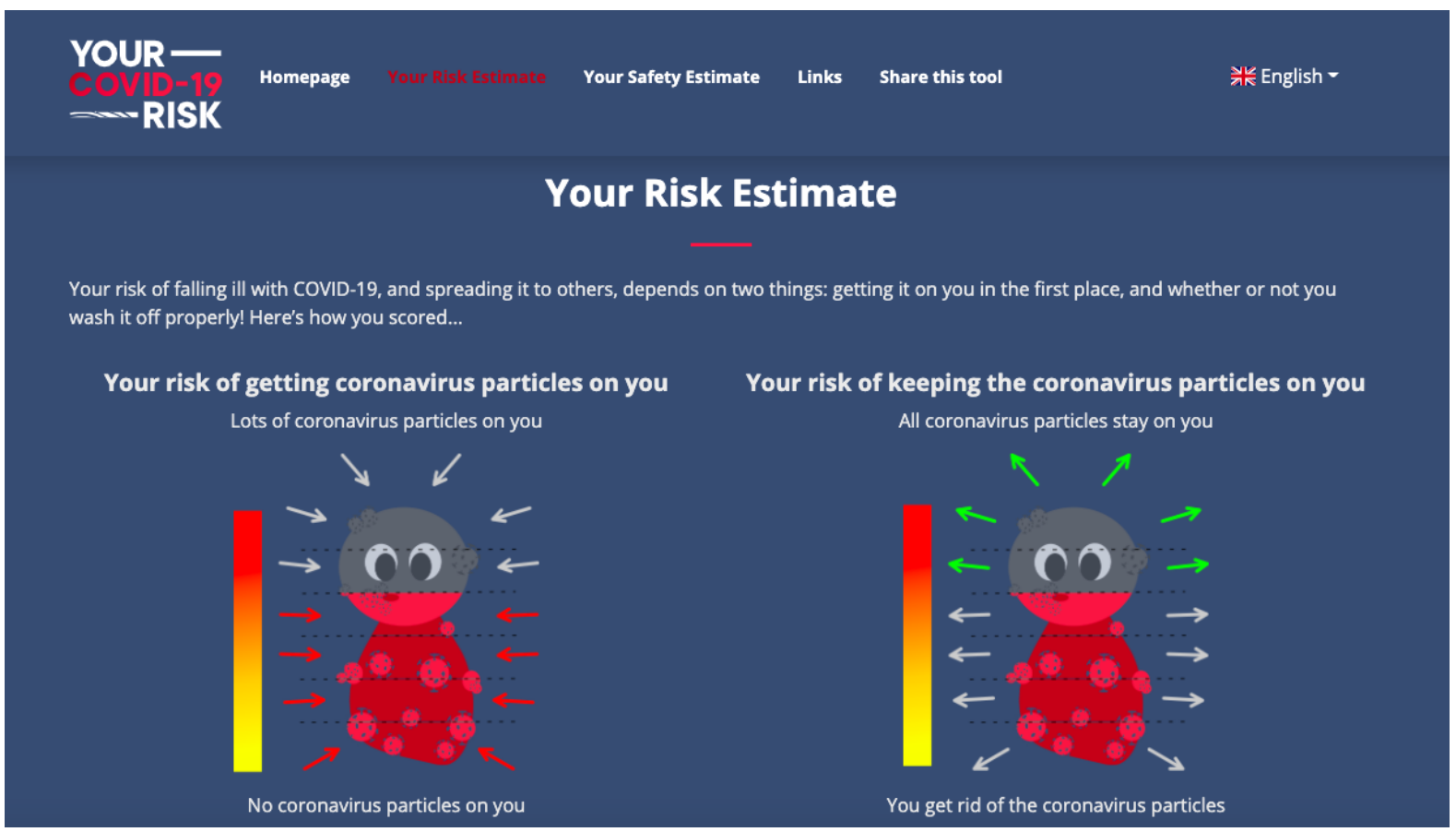

Figure 3: An example of the graphic representation of risk estimates.

\section{Designing the intervention}

The intervention was developed using Acyclic Behaviour Change Diagrams (ABCDs) to optimise alignment between the aspects of human psychology that it targets, the behaviour change principles that were used, and the adequate application of those behaviour change principles. This alignment ensures that the tool offers users what is needed for behaviour change based on a causal-structural chain comprising seven links. The full causal-structural chain of behaviour change is as follows: (1) An effective behaviour change principle is used, and (2) according to the corresponding conditions for effectiveness, (3) the behaviour change principle is applied into an application that targets (4) an 
important and sufficiently specific sub-determinant, which is a part of (5) a generally defined determinant that determines (together with other determinants), (6) a subbehaviour, which is a specifically defined behaviour that forms (together with other subbehaviours), (7) the target behaviour. To illustrate how ABCDs informed the tool development, this concept of the causal-structural chain will first be explained.

The chain ends with enactment of the target behaviour. Such target behaviours are typically broadly defined, such as social distancing, self-isolation or hand-washing. Each of these behaviours consists of sub-behaviours. For example, effective hand-washing requires using soap, cleaning hands for at least 20 seconds, and using the right technique. To the degree that each sub-behaviour is determined by individuals' psychology (as opposed to their environments; together these, by definition, determine behaviour), these influences can be captured in a set of determinants: the psychological constructs that theoretically determine the individual's behaviour. Determinants can be defined generally (such as 'attitude') or at a more specific sub-level (sub-determinants such as 'perceived probability that using soap will remove the virus from my hands'). Although most psychological research occurs at the general level (that of determinants), intervention providers and health promoters need to communicate specific messages (on the level of sub-determinants). ABCDs therefore distinguish both construct levels.

The first three links for the causal-structural chain are the behaviour change principles, the corresponding conditions for effectiveness, and the applications in which the principles are applied. To successfully change people's psychology -i.e., (sub-)determinantsrequires correct application of behaviour change principles: techniques that leverage the evolutionary learning processes through which humans learn ${ }^{22}$. Like determinants, those behaviour change principles are defined at a general, abstract level, and have to be 
translated into specific practical applications for use in interventions. In this translation, the intervention developer needs to keep each behaviour change principle's conditions for effectiveness in mind: the parameters within which an application can still feasibly engage the underlying evolutionary learning processes from which the behaviour change principle derives its effectiveness ${ }^{22}$. One application can contain several behaviour change principles and the same behaviour change principle can be applied in various manifestations in different applications, depending on the available intervention medium, the target population's culture and other characteristics, and what is known to work best in a given context or for a given behaviour or population. This can quickly become confusing, and $\mathrm{ABCDs}$ can facilitate retaining an overview of which behaviour change principles are implemented in which applications and how the conditions for effectiveness are safeguarded. 


\section{Acyclic Behavior Change Diagram}

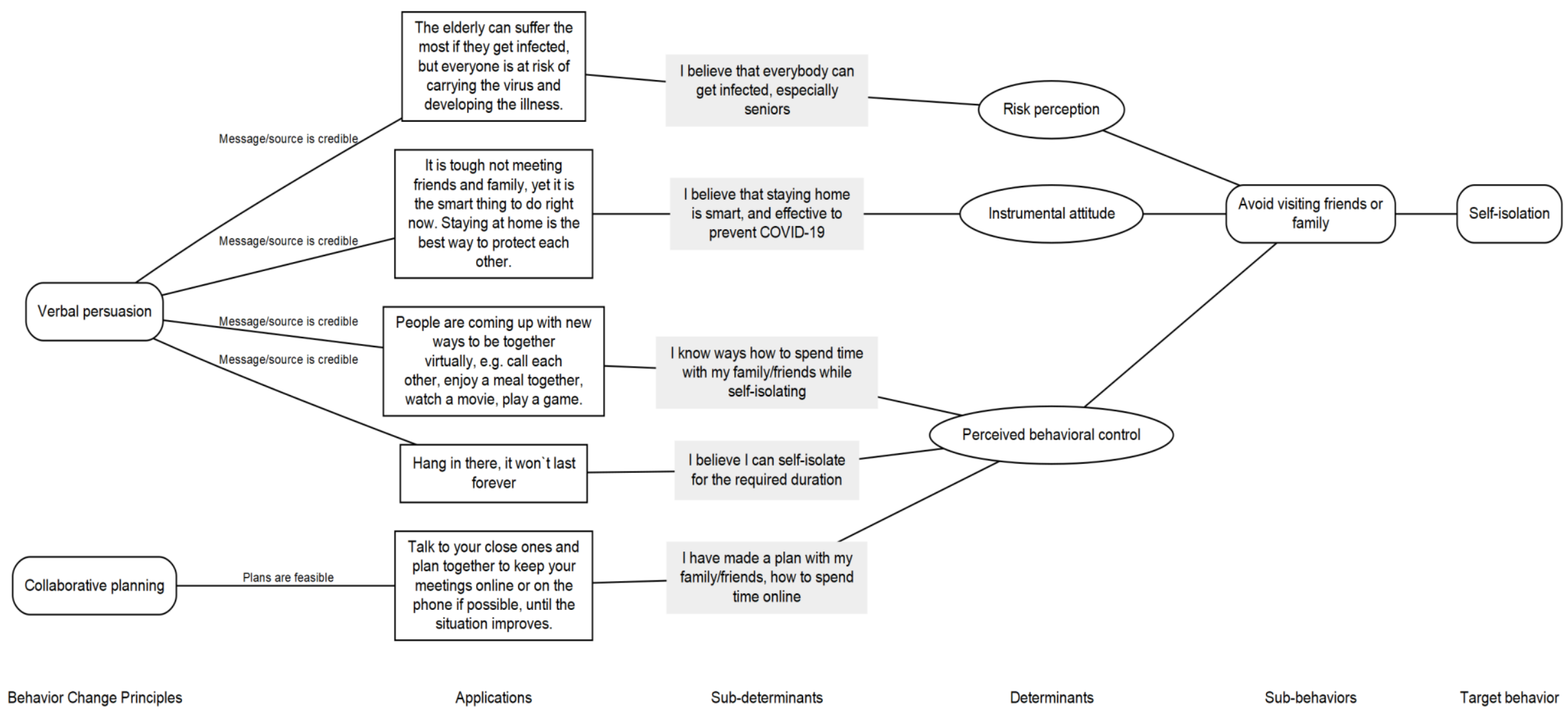

Figure 4: An illustrative fragment of one of the Acyclic Behaviour Change Diagrams used to develop the intervention 
$\mathrm{ABCDs}$ are built from matrices that are commonly stored in spreadsheets where every row represents one causal-structural chain. An ABCD matrix expresses the assumptions underlying the expected effectiveness of any health communication, intervention or campaign. These matrices can be parsed and used to produce the ABCDs (i.e., the diagrams), which are visual representations of the causal-structural chains. A fragment of one of the ABCDs underlying the tool's intervention is shown in Figure 4, specifically the part of the intervention targeting social isolation in participants who indicated that they still leave the house to visit friends and family. Please note that the context of the intervention (low resources to develop applications; need for applications to be suitable for various countries) limited the possibilities of applications to mostly being text-based messages. ABCDs express exactly which (sub-)determinants an intervention addresses and which behaviour change principles the intervention developers aim to use to change the (subdeterminants. As such, ABCDs make it possible to spot errors, discuss the choices that were made, and facilitate the process of iteratively improving the tool over time based on new insights about important (sub-)determinants (as well as adaptation of the intervention by others). As we learn more about why people do what they do in terms of COVID-19-related behaviours, we are able to improve the tool to better meet people's needs. To enable these improvements, we integrated another important aspect into the tool, namely the Determinant Mapping Questions.

\section{The Determinant Mapping Questions}

When we started on the ABCDs (i.e., started compiling what was known about the most relevant sub-behaviours to distinguish and which sub-determinants and determinants 
were the most important predictors of each sub-behaviour), it became apparent that almost no information was available on the determinant structures of the target behaviours. Given that the upper bound for an intervention's effectiveness is formed by how many of the relevant determinants are successfully changed, to improve the tool, we would need to learn about the target behaviours' determinant structures. We asked tool users whether they would be willing to help improve the tool, and if they were, to answer additional questions about (sub-)determinants, specifically regarding keeping distance from others (we used country-specific guidelines, e.g., 1.5 meters in the Netherlands, using the World Health Organization (WHO) recommendation of 1 meter for countries where country-specific recommendations were unavailable). However, we needed these (sub-)determinants to be standardised across languages as much as possible, without the resources to conduct crosscultural validation studies. As a solution we used a technology called Decentralized Construct Taxonomies.

Decentralized Construct Taxonomies (DCTs) were designed to address hidden heterogeneity in construct definitions and operationalisations in psychological science ${ }^{13}$. Such heterogeneity manifests, for example, in the diversity of symptoms measured by depression scales ${ }^{23}$, low convergence of theory of mind measures ${ }^{24}$, problematic overlap in emotion measures ${ }^{25}$, and confusion about the nature and measurement of self-efficacy relative to other determinants ${ }^{26}$. Brick et al. recently proposed that a 'psychological essentialistic bias' contributes to overreliance on the assumption that psychological constructs are natural kinds ${ }^{27}$, an assumption that is questioned with some regularity ${ }^{28-31}$, yet seems hard to eliminate. DCTs provide a tool to make the exact construct definition that is used in a given study explicit and bind that specific definition to instructions for 1) developing measurement instruments for that construct; 2) coding measurement 
instruments (as used in systematic reviews); 3) eliciting construct content; and 4) coding qualitative data as informative about a construct (both of which are used in qualitative research).

First translating a set of DCT specifications for all determinants that we planned to ask tool users about enabled prompting convergence of people's hitherto implicit 'personal' construct definitions. However, as DCT specifications derive their value from their comprehensiveness, these could not be developed in the scope of this project. Therefore, we limited the Determinant Mapping Questions to constructs for which we already had a set of DCT specifications available: those defined in the Reasoned Action Approach (RAA).

The RAA is a theoretical framework that addresses reasoned action (i.e., planned behaviour) and behaviour change. In line with the RAA, attitudes towards the behaviour, perceived norms, and perceived behavioural control determine individuals' intentions, while intentions predict human behaviour. The authors of this framework have extensively described how to measure its specific components and constructs ${ }^{12}$. These descriptions have been further specified in a set of DCTs that were used in this project.

Based on these DCTs, a set of template questions was created in English and then translated from English to each of the 22 languages (and back-translated as volunteer capacity allowed). In parallel with this process, lacking the resources to conduct qualitative research to elicit construct content, we 'self-elicited' a long-list of potential subdeterminants. This long-list was then curated in five iterative steps to remove duplicates and complement sub-determinants consistent with the RAAs symmetrical construct definition structure (e.g., when an experiential attitudinal expectation was suggested, the corresponding experiential attitudinal expectation was added to the list of questions). The final list contained 276 questions (available through the associated repository); however, due 
to cultural differences, we allowed translating teams to decide if any question had to be excluded as it was considered sensitive or nonsensical in the local context. The group of volunteers then applied the calibrated question templates from the previous step to these questions to achieve a set of translated questions that, as much as possible, measured the same constructs. These processes (translation of the template questions, construct content elicitation and curation, and translation of the final set of questions) were conducted using Google Sheets, enabling real-time collaboration. These spreadsheets were then imported into $\mathrm{R}$ for further processing.

\section{Project architecture and applications}

This project was possible because of a large number of Free/Libre and Open Source Software (FLOSS) solutions in combination with free resources graciously offered by LimeSurvey Gmbh, Netlify, and Slack. We only wanted to use FLOSS because we wanted the product of the volunteer effort invested in this project to be freely available for everybody. For example, had we not implemented these Open Science principles and used proprietary software for central components of the project, these products would have been virtually inaccessible to organisations and individuals that were not well funded. In addition, this project had no funding itself, and although many of our volunteers could access proprietary software through their employers, the voluntary nature of their participation meant using employer resources was not a straightforward option.

In composing the so-called 'stack' or 'application architecture' for this project (i.e., the layers of software applications that implement the functionalities that we require), we needed to resolve a core project asymmetry. On the one hand, we needed to collaborate on the development of a system with many interlinking components in many languages. On the other hand, we had a large group of volunteers who were familiar with basic office software 
such as spreadsheets, but less with version control and collaboration solutions such as Git and programming languages such as R, PHP or JavaScript. As these volunteers would produce and translate a large amount of content in a complex data model, we solved it in the following way.

First, we chose two free but proprietary tools to support our internal communication and facilitate collaborating on the same documents. We did not consider this problematic because these tools mostly supported ephemeral interactions, and to the degree that we would need to retain data or products, these could be exported to open formats. Specifically, the first tool we heavily relied on was Slack, an asynchronous communications hub. This enabled us to communicate in sub-groups of arbitrary size and allowed all volunteers to read along when they so desired. The threaded communication format kept the interaction relatively organized (the sheer number of messages was still considered overwhelming by many volunteers, but our distributed roles meant that no single person needed to read everything). The second tool was Google Docs, an online synchronous collaboration office suite, of which we used the word processor application (also called Google Docs) and the spreadsheet application (called Google Sheets). We used the approximately 130 Google Docs documents to provide instructions to the team of volunteers so that their actions were streamlined and different subgroups could work in parallel. We used Google Sheets as a stand-in for a relational database. Whenever structured data needed to be generated, or whenever something needed to be translated, we used Google Sheets for this, with carefully structured spreadsheets that had one worksheet for each language. Because Google Sheets has an Application Programming Interface (API) we were able to directly import these data into R.

Second, we used Git to collaborate on the project files with the software developers, designers, and data scientists in the project team. Git is a version control and collaboration solution that has quickly become the standard for software developers and is also becoming 
more commonplace among scientists: it can be thought of as the tracked changes functionality common in word processors such as LibreOffice Writer or Microsoft Word, but for all files, and with many more options. We used GitLab, a FLOSS development and operations platform augmenting Git's functionality allowing us, among other things, to effortlessly host a website with the rendered R Markdown files produced by the project. This Git repository also included the main website of the project, which was written mostly by one of our volunteers in HTML, CSS, and JavaScript, using the Bootstrap framework and the jQuery and i18next JavaScript libraries. This static website consisted partly of JavaScript Object Notation (JSON) files with content that was produced by R scripts that imported the raw data from Google Sheets.

We used LimeSurvey ${ }^{17}$ as an engine to present the tool users with questions and compute their risk estimates. Because the LimeSurvey survey had 543 questions organised in 156 question groups, and all text was translated from English into 21 languages ( 22 total), it was not feasible to manually enter and update the thousands of text elements (questions, answer options, etc) for each language (e.g., the survey contained 56298 answer options in total). One of us therefore developed an R package called \{limonaid \} that we used to programmatically produce a file that LimeSurvey could import. This file was again produced based on processing of data that were imported from Google Sheets. For readers who want to import the file into their LimeSurvey instance: note that because the file is 17.6 megabytes, importing it will likely require updating the server settings in LimeSurvey itself or in PHP or Apache.

Another core component was $\mathrm{R}^{32}$, which we used to parse all data and content from the spreadsheets with translations. This effectively means that the data model governing those spreadsheets was hardcoded into the R Markdown source code files. We had R Markdown files to perform the required analyses for the two expert consultations and to compute the 
Risk Model, to produce 14-day case numbers for each country to assign country-level risk, to produce the ABCDs that formed the intervention, to prepare the JSON files for the static website, to produce the survey file to import into LimeSurvey, and to analyse the tool users' answers (e.g., generating CIBER plots). 


\section{References}

1. Gates, B. Responding to Covid-19—a once-in-a-century pandemic? N. Engl. J. Med. 382, 1677-1679 (2020).

2. Xiao, Y. \& Torok, M. E. Taking the right measures to control COVID-19. Lancet Infect. Dis. 20, 523-524 (2020).

3. Hellewell, J. et al. Feasibility of controlling COVID-19 outbreaks by isolation of cases and contacts. Lancet Glob. Health (2020).

4. West, R., Michie, S., Rubin, G. J. \& Amlôt, R. Applying principles of behaviour change to reduce SARS-CoV-2 transmission. Nat. Hum. Behav. 4, 451-459 (2020).

5. Van Bavel, J. J. et al. Using social and behavioural science to support COVID-19 pandemic response. Nat. Hum. Behav. 1-12 (2020).

6. Bonell, C. et al. Harnessing behavioural science in public health campaigns to maintain 'social distancing'in response to the COVID-19 pandemic: key principles. J Epidemiol Community Health (2020).

7. Zhang, X. Epidemiology of Covid-19. N. Engl. J. Med. 382, (2020).

8. Kwasnicka, D. et al. Promoting scientific integrity through open science in health psychology: results of the Synergy Expert Meeting of the European health psychology society. Health Psychol. Rev. 1-35 (2020).

9. Beyene, K., Chan, A. H. Y., Green, J. A. \& Hermsen, S. Your Covid-19 Risk: Reflections on the Development of the Tool. Health Psychol. Bull. 5, 61-69 (2021).

10. Sachs, J. D. et al. The Lancet COVID-19 Commission. The Lancet (2020).

11. Peters, G.-J. Y. \& Crutzen, R. The core of behavior change: introducing the Acyclic Behavior Change Diagram to report and analyze interventions. https://osf.io/w85va (2021) doi:10.31234/osf.io/w85va. 
12. Fishbein, M. \& Ajzen, I. Prediction and change of behavior: The reasoned action approach. (Taylor \& Francis Group, 2010).

13. Peters, G.-J. Y. Decentralized Construct Taxonomies. https://osf.io/xebhn (2020) doi:10.31234/osf.io/xebhn.

14. Crutzen, R., Peters, G.-J. Y. \& Noijen, J. Using Confidence Interval-Based Estimation of Relevance to Select Social-Cognitive Determinants for Behavior Change Interventions. Front. Public Health 5, 165 (2017).

15. Peters, G.-J. Y. \& Crutzen, R. Establishing determinant importance using CIBER: an introduction and tutorial. Eur. Health Psychol. 20, 485-495 (2018).

16. Bruin, M. de. Behavioural perspective on the transition from the COVID-19 pandemic to an endemic phase. (2021) doi:10.31234/osf.io/kun3j.

17. LimeSurvey Project Team / Carsten Schmitz. LimeSurvey: An Open Source survey tool. http://limesurvey.org (2020).

18. CBS. ICT-gebruik huishoudens - ICT, kennis en economie|2018. ICT, kennis en economie | 2018 https://longreads.cbs.nl/ict-kennis-en-economie-2018/ict-gebruikhuishoudens.

19. Telemedia. Market Snapshot: Desktop and mobile internet usage in 2020 | Platforms \& Technology. Telemedia Online https://www.telemediaonline.co.uk/market-snapshotdesktop-and-mobile-internet-usage-in-2020/ (2020).

20. Mitter, S. Indian internet is more rural, local, and mobile-first than ever. YourStory.com https://yourstory.com/2020/05/half-billion-active-users-indian-internetrural-local-mobile-first (2020).

21. Knittle, K. P., Peters, G.-J. Y., Heino, M. T. J., Tobias, R. \& Hankonen, N. Potential for change $(\mathrm{P} \Delta)$ : New metrics for tailoring and predicting response to behavior change interventions. (2019) doi:10/ghqmg3. 
22. Crutzen, R. \& Peters, G.-J. Y. Evolutionary learning processes as the foundation for behaviour change. Health Psychol. Rev. 12, 43-57 (2018).

23. Fried, E. I. The 52 symptoms of major depression: Lack of content overlap among seven common depression scales. J. Affect. Disord. 208, 191-197 (2017).

24. Warnell, K. R. \& Redcay, E. Minimal coherence among varied theory of mind measures in childhood and adulthood. Cognition 191, 103997 (2019).

25. Weidman, A. C., Steckler, C. M. \& Tracy, J. L. The jingle and jangle of emotion assessment: Imprecise measurement, casual scale usage, and conceptual fuzziness in emotion research. Emotion 17, 267-295 (2017).

26. Williams, D. M. \& Rhodes, R. E. The confounded self-efficacy construct: review, conceptual analysis, and recommendations for future research. Health Psychol. Rev. 10, 113-128 (2016).

27. Brick, C., Hood, B., Ekroll, V.\& de-Wit, L. Illusory essences: A bias holding back theorizing in psychological science. https://osf.io/eqma4 (2020) doi:10.31234/osf.io/eqma4.

28. Peters, G.-J. Y. \& Crutzen, R. Pragmatic nihilism: how a Theory of Nothing can help health psychology progress. Health Psychol. Rev. 11, 103-121 (2017).

29. Peters, G.-J. Y. \& Crutzen, R. Confidence in constant progress: or how pragmatic nihilism encourages optimism through modesty. Health Psychol. Rev. 11, 140-144 (2017).

30. Fried, E. I. What are psychological constructs? On the nature and statistical modeling of emotions, intelligence, personality traits and mental disorders. Health Psychol. Rev. 11, 130-134 (2017).

31. Gruijters, S. L. K. The reasoned actions of an espresso machine: a comment on Peters and Crutzen (2017). Health Psychol. Rev. 11, 125-129 (2017). 
32. R Core Team. R: A Language and Environment for Statistical Computing. (R Foundation for Statistical Computing, 2020). 


\section{Data availability}

Consistent with the Open Science principles to make all materials, source code, analysis scripts, and data freely available, everything produced in this project is licensed under Creative Commons Attribution licenses or similarly, and available from the project's GitLab repositories https://gitlab.com/a-bc/your-covid-19-risk and https://gitlab.com/abc/your-covid-19-risk-data. The files in both repositories are also available through the project's main Open Science Framework repository available at https://osf.io/vkdyt/. The frozen registrations in component projects will retain the state of these files regardless of whether the Git repository is updated in the future.

\section{Acknowledgements}

The project was initiated by the Academy of Behaviour Change (https://a-bc.eu), a Dutch non-profit foundation contributing to the theory, methodology, application, and knowledge translation of behaviour change science, to support prevention organisations to develop effective behaviour change interventions, campaigns, and programs. The tool was developed by over 150 volunteers, most of them listed as authors, who contributed their time and expertise to develop, implement, and disseminate the tool internationally.

\section{Author contributions}

All authors contributed to writing by reviewing and editing (CRediT category 'Writing - review \& editing'). Gjalt-Jorn Peters and Sylvia Roozen conceived of the project (CRediT category 'Conceptualization'). Dominika Kwasnicka and Gjalt-Jorn Peters wrote the original draft (CRediT category 'Writing - original draft'). Other author contributions were as follows (in alphabetic order and mapped onto the CRediT taxonomy): Mahdi Algargoosh 
contributed to data curation \& resources; Eskinder Eshetu Ali contributed to resources; Mudassir Anwar contributed to data curation, project administration \& resources; Sali Rahadi Asih contributed to data curation, project administration \& resources; Zuhal Feryal Baltas contributed to project administration \& resources; Emma Berry contributed to data curation, formal analysis \& project administration; Kebede Abera Beyene contributed to project administration \& resources; Katarzyna Anna Campbell contributed to resources; Bruno Moreira Carneiro contributed to data curation; Laura Castillo-Eito contributed to data curation, project administration \& resources; Amy Hai Yan Chan contributed to data curation, formal analysis, project administration, resources \& supervision; Sabrina Cipolletta contributed to data curation, project administration, resources \& supervision; Rik Crutzen contributed to resources \& supervision; Ann DeSmet contributed to data curation, formal analysis, project administration \& resources; Triana Kesuma Dewi contributed to data curation, project administration \& resources; Alexandra Lelia Dima contributed to data curation, project administration \& resources; Jorge Encantado contributed to project administration \& resources; Tracy Epton contributed to formal analysis \& project administration; João Melo Figueiredo contributed to software; Gustavo DalCin Fracaroli contributed to data curation; Gebremedhin Beedemariam Gebretekle contributed to data curation, project administration \& resources; Pierre Gérain contributed to data curation, project administration, resources \& supervision; Cristina Albuquerque Godinho contributed to project administration \& resources; Lisa Graham-Wisener contributed to project administration \& resources; James A. Green contributed to data curation, formal analysis, project administration, resources, software \& visualization; Jenny M Groarke contributed to data curation, formal analysis \& project administration; Thomas Gültzow contributed to data curation, project administration \& resources; Elif Basak Guven contributed to project 
administration \& resources; Roel C.J. Hermans contributed to data curation, project administration \& resources; Jennifer Inauen contributed to project administration, resources \& supervision; Angelos P. Kassianos contributed to project administration \& resources; Tatiana Valerievna Kazantseva contributed to resources; Els Keyaerts contributed to data curation; Laura Maria König contributed to data curation, project administration \& resources; Dominika Kwasnicka contributed to data curation, project administration, resources \& supervision; Daniela Lange contributed to data curation \& resources; Emelien Lauwerier contributed to data curation, project administration \& resources; Yongchan Lie contributed to project administration \& resources; Andrian Liem contributed to data curation, project administration \& resources; Aleksandra Luszczynska contributed to data curation \& resources; Marta M. Marques contributed to data curation, project administration \& resources; Hannah Catherine Moore contributed to data curation; Chris Noone contributed to data curation, project administration \& resources; Johanna Nurmi contributed to data curation, project administration \& resources; Ratri Nurwanti contributed to data curation, project administration \& resources; Elif Suna Ozbay contributed to project administration, resources \& supervision; Iga Palacz-Poborczyk contributed to data curation, project administration \& resources; Rebecca Anne Pedruzzi contributed to data curation, formal analysis, project administration, resources, software \& supervision; Gjalt-Jorn Ygram Peters contributed to formal analysis, project administration, resources, software, supervision \& visualization; Louise Poppe contributed to data curation, project administration \& resources; Lucy Mabel Porter contributed to data curation, formal analysis, project administration \& resources; Daniel Powell contributed to formal analysis, project administration \& resources; Bruna Salati Nani Rinaldi contributed to data curation, formal analysis, project administration \& resources; Sylvia Roozen contributed to data curation, 
formal analysis, project administration, resources, software \& supervision; Alexis Ruffault contributed to project administration \& resources; Carsten Schmitz contributed to resources \& software; Urte Scholz contributed to project administration \& resources; Ana-Maria Schweitzer contributed to data curation, formal analysis, project administration \& resources; Yasemin Selekoglu Ok contributed to data curation, project administration \& resources; Medha Shree contributed to data curation, formal analysis \& resources; Yasinta Astin Sokang contributed to data curation, formal analysis, project administration \& resources; Mei Yee Tang contributed to data curation \& resources; Gill A ten Hoor contributed to data curation, project administration, resources \& supervision; Silvia Caterina Maria Tomaino contributed to project administration; Tugce Varol contributed to project administration \& resources; Stefan Verweij contributed to resources \& software; Stan Vluggen contributed to data curation, project administration, resources \& supervision; Lisa Marie Warner contributed to data curation, formal analysis \& resources; Rochelle E Watkins contributed to data curation, project administration \& resources; Szilvia Zörgo contributed to project administration \& resources.

\section{Competing interests}

The authors have no competing interests to disclose. 